RESEARCH PAPER

\title{
"Start to stop": results of a randomised controlled trial of a smoking cessation programme for teens
}

\author{
L A Robinson, M W Vander Weg, B W Riedel, R C Klesges, B McLain-Allen
}

Tobacco Control 2003;12(Suppl IV):iv26-iv33

See end of article for authors' affiliations ....................

Correspondence to: Leslie A Robinson, PhD,

The University of Memphis Department of Psychology, 202 Psychology Building, Memphis, TN, 381523230, USA; L.Robinson@ mail.psyc.memphis.edu

\begin{abstract}
Objective: To examine the feasibility, acceptability, and effectiveness of a school based smoking cessation programme among students caught smoking at school.

Design: A randomised controlled trial comparing cessation rates among students in a behavioural cessation programme and those receiving self help materials only.

Setting: Eighteen schools in the Memphis, Tennessee area.

Subjects: Two hundred and sixty one adolescent cigarette smokers (166 male, 95 female) averaging 15.8 years of age.

Intervention: Students assigned to the intervention received a four session behavioural treatment programme administered individually by a health educator. In addition, these students received stage matched intervention in brief phone calls monthly until the one year follow up.

Main outcome measure: Self reported and biochemically verified smoking cessation at post-test and 12 month follow up.

Results: Recruiting students who were caught smoking at school proved to be highly successful. Participants rated the programme favourably, and retention rates were high. Although treated participants improved more in tobacco related knowledge relative to controls $(p=0.002)$, there were no group differences in changes in attitudes toward smoking. In addition, treated and control participants demonstrated no significant differences in cessation rates both at post-test and follow up. Comparisons between self reported cessation rates and those obtained under bogus pipeline conditions or with biochemical verification suggested significant falsification of cessation among participants.

Conclusions: Our results failed to demonstrate any significant effect of the cessation programme on smoking rates for treated adolescents compared with controls. Our findings also highlight the importance of utilising strong methodology in research on adolescent smoking cessation, including control groups and biochemical verification of smoking status.
\end{abstract}

$\mathrm{D}$ espite decades of prevention efforts, smoking rates among adolescents remain unacceptably high. At present, almost $36 \%$ of high school students are current smokers, ${ }^{1}$ and the negative health effects of tobacco use begin for these youth even in adolescence. ${ }^{2}$ Further, those who smoke in young adolescence are 16 times more likely to become adult smokers. ${ }^{3}$ If present tobacco use trends continue, it has been projected that five million individuals 17 years old or younger will become smokers and die prematurely of a smoking related illness in adulthood. ${ }^{4}$

Fortunately, teen smokers do seem to recognise the dangers of smoking. Approximately $75 \%$ of high school smokers have made at least one quit attempt, ${ }^{5}$ and in one cross sectional study of 12 th grade smokers, $31 \%$ had attempted to quit more than once, with $13 \%$ reporting three or more attempts. ${ }^{6}$ Contrary to common lore, these efforts often appear to be prompted by teens' worries about their health. ${ }^{78}$ In fact, concern about future health has been identified as the single most frequently reported motivator for teens' quit attempts, and concern regarding current health is the second most common reason. ${ }^{9}$

Despite this motivation, teen smokers' attempts to quit are hampered by at least two major factors. First, adolescents seem to have difficulty developing a coherent plan for quitting. Certainly, studies of the methods used by adolescents to quit smoking suggest that teens lack awareness of many of the strategies used successfully by adults. ${ }^{10}{ }^{11}$ For example, in one study $81 \%$ of adolescents who had attempted quitting did not even try to avoid exposure to smoke during the quit attempt. ${ }^{12}$ The second issue involves withdrawal.
Even among adolescent smokers whose consumption rate is relatively low, withdrawal symptoms can be quite significant. Half of all adolescent smokers who try to quit report withdrawal symptoms, ${ }^{13}$ and it has been estimated that one to three out of five adolescent smokers is dependent on nicotine. ${ }^{14}$

Not surprisingly, then, the odds that adolescent smokers will quit unaided are very low. In a recent review, Sussman ${ }^{15}$ reported that over a 3-5 month interval, spontaneous cessation rates among teen smokers range from $0-11 \%$. The few studies using one year follow ups have observed somewhat larger cessation rates, ranging from $12 \%{ }^{16}$ to $21 \%{ }^{13}$ However, researchers examining even longer time intervals have obtained lower estimates. For instance, McNeill found that only $3 \%$ of daily smokers quit during a two year follow up, ${ }^{17}$ and a spontaneous abstinence rate of 5.3\% was observed for adolescent smokers followed for three years. ${ }^{11}$

Given that teens want to quit, and that they have little success on their own, it is surprising how little research has been aimed at developing and evaluating smoking cessation programmes for teens. In a 1999 review, Sussman and colleagues $^{18}$ identified fewer than 20 studies of quit programmes for adolescents. They concluded that such programmes yielded quit rates of $21 \%$ at post-test and $13 \%$ at follow up. However, the studies under review were fraught with methodological problems. For example, few included control groups, and smoking status was rarely assessed with a bogus pipeline, much less any form of biochemical verification. Follow ups were short, and only two studies 
tracked participants for a year or more. Notably, studies averaged only 86 smokers. Retention was also troubling; on average, only $77 \%$ of the enrolees provided post-test data. Further, few studies addressed "programme reach", or the extent to which the programme under evaluation successfully attracted adolescent smokers. This issue is important, because even if programmes are effective, if they can only induce a few teens to participate, they will have limited impact on the broader problem of adolescent tobacco addiction.

Although interest in helping teens quit has increased since the publication of the Sussman review, the field has struggled to develop effective cessation programmes for adolescents. Recently, three well controlled evaluations of treatment programmes for adolescent smokers all failed to find signi ficant differences between treated teens and controls. ${ }^{19-21} \mathrm{~A}$ fourth study obtained results that appeared more promising at first glance. ${ }^{22}$ In this investigation, 59\% of the treated teens were identified as abstinent at post-test, compared with $17 \%$ of controls. However, closer inspection of this study suggests some problems in the measurement of abstinence. To be defined as "quit", participants had to obtain expired air carbon monoxide (CO) of $<5$ parts per million (ppm). Nonetheless, the mean salivary cotinine values reported among quitters suggest that many defined as abstinent (by CO values) must have been smoking. Thus, even these positive outcomes may have been overestimated.

Sussman and colleagues ${ }^{23}$ recently completed a large controlled evaluation of a smoking cessation programme. In this study, 259 adolescent volunteers were assigned to treatment, and another 78 teens were recruited as controls. At post-test, $17 \%$ of the treated students had quit, compared with only $8 \%$ of the controls. However, interpretation of these findings is complicated because the youth were apparently not randomly assigned to groups. Further, the rates of dropouts and students lost to follow up were quite high, and the reach of the programme was limited.

In summary, few cessation programmes targeting adolescents have been developed and evaluated, and the investigations that have been conducted have suffered from serious methodological flaws. Control groups have rarely been included, and student self reports of smoking status have often remained unverified. Follow ups tend to be short, and dropout rates are generally high. Further, the reach of teen smoking cessation programmes has been problematic. Teens usually want to keep their smoking habit a secret from their parents, and the required consent procedures have made it difficult to enrol participants. Thus, the students who most need help in quitting may be least likely to seek it.

Our programme took a different tact in seeking eligible adolescent smokers. Rather than soliciting volunteers, we offered the programme to youth who had been caught smoking in school. The advantage of this method is that the behaviour was already public; thus, students did not have to avoid treatment because it would disclose their habit. Further, most students found being caught for smoking to be embarrassing, and they felt increased pressure to quit. As an added incentive, all but one of the schools in this investigation reduced punitive sanctions (for example, suspension) for students who enrolled in the research protocol, regardless of whether they were in the treatment or control arm. Thus, we caught students at a "teachable" moment, in which many of the natural barriers to treatment were removed, and some extra incentives were in place. Our goals were to test the feasibility of placing a cessation programme in a high school environment, evaluate the acceptability of the curriculum to the students, and determine whether participation in the programme significantly improved quit rates relative to a control.

\section{METHODS}

Participants were students aged 13-19 years, recruited from 18 schools in the Memphis, Tennessee, area. They were typically referred to the study by school administrators for violating policies regarding tobacco possession at school. A few students were self referred or sent by their parents. There was no minimal smoking rate required for entry into the study.

Of the 381 students who were offered participation in the research, $316(83 \%)$ agreed to enrol. Of these 316 , the data for 33 participants in the overall study were not yet available for inclusion in the current analyses. Of the remaining 283 students who entered the trial, only 22 dropped out before post-test $(8 \%)$. Thus, the retention rate through post-test was $92 \%$. In addition, 150 participants (91\% of those eligible to date) have finished the 12 month follow up. (The follow up phase of the project is still ongoing.) Those who declined to take part in the study did not differ from the 261 study participants in age, gender, ethnicity, or parental education (all p's > 0.10). The remainder of the paper will focus only on participants who completed the post-treatment protocol.

\section{Procedure}

Participants were randomly assigned to a four session behavioural smoking cessation programme or to a control condition. The level of assignment was by participant within schools; thus, the unit of assignment was the student rather than the school. Two thirds of students were assigned to treatment. This procedure was much more acceptable to the school system, which preferred to maximise treatment opportunities. At the same time, it still allowed for a randomised control with sufficient statistical power for our purposes.

At entry to the study, participants completed a paper-andpencil baseline survey. A post-test was administered approximately three weeks later, a date that corresponded with the completion of the behavioural programme. Over the next year, students in both groups received monthly phone calls to assess smoking status. Those assigned to the treatment programme were called by the health educator who had worked with them in their intervention programme. During the call, the health educator also delivered a brief stage based intervention (see below). Twelve months after post-test participants completed a follow up phone assessment. For those who reported abstinence at the one year follow up, biochemical verification of smoking status was arranged.

All of these assessments were conducted by trained members of our research team (that is, either research assistants or health educators). Except in a very few cases that presented extreme logistic difficulties, post-test and follow up data were collected only by staff members who had not been involved in a given student's treatment programme. This plan was designed in order to avoid making students feel pressured to report false positive outcomes to their previous health educator. Of course, the biochemical verification procedures also helped to ensure more accurate reporting.

\section{Interventions}

\section{Behavioural intervention}

The Start to Stop (STS) programme was designed using a social influence theoretical framework, in which youth were assumed to need first motivational enhancement, and second, training in the social and stress management skills necessary to complete a successful quit attempt. A total of four weekly 50 minute individual sessions with a health educator were held. The curriculum contained two primary components. The first used the principles of motivational interviewing to enhance motivation to quit. ${ }^{24}$ Discussions centred around topics such as the actual prevalence of 
smoking in teens, the attitudes of adolescents toward smoking, beliefs about the "benefits" of smoking, cigarette advertising techniques, the short term health effects of smoking, and its monetary costs.

The second component of the programme was designed to provide teens with tools for quitting. Participants were taught standard behavioural techniques, including setting a quit date, stimulus control, developing coping skills, enlisting social support, reducing weight gain, and relapse prevention.

In addition to the initial four sessions, a brief intervention based on the stages of change model ${ }^{25}$ was administered during each of the monthly follow up phone calls. Students were first queried about their smoking status and intentions to quit and then given brief stage matched messages to enhance their motivation for quitting.

\section{Control intervention}

Participants assigned to the control condition received the "I QUIT" pamphlet provided by the Centers for Disease Control and Prevention. The pamphlet consists of suggestions for quitting smoking, including many of the behavioural strategies outlined above. However, students in the control condition did not receive any individual instruction.

\section{Health educators}

Two health educators served as interventionists. One had a Bachelor of Arts degree and had served for many years as a teacher and a nurse. The second health educator had a Master's degree, with extensive experience as a substance abuse counsellor. Their training for this project began with a two day formal training programme in motivational interviewing delivered by a certified expert. To verify their competency both in motivational interviewing and in the content of the cessation programme, the health educator practised the entire programme with team members under the direct supervision of the grant manager, a clinical psychologist also trained in motivational interviewing. Only after it was established that they had been trained to criterion were the health educators allowed to begin the programme with students.

\section{Fidelity checks}

After the programme had begun, periodic checks were made to ensure that the health educators had not "drifted" from the manualised intervention. Independent research assistants observed treatment sessions for randomly selected students, and a checklist was used to ensure that health educators were delivering the essential components of the programme as prescribed.

\section{Measures}

\section{Cigarette smoking}

Participants were asked to report the number of years since their first cigarette, whether they had smoked 100 or more cigarettes in their lifetime, and the frequency of quit attempts in the past year. Students were also asked which of the following most accurately reflected their current consumption: I have smoked (a) only one cigarette or a few cigarettes just to try, (b) less than one cigarette per month, (c) about 1 to 3 cigarettes per month, (d) about 1 to 6 cigarettes per week, (e) about 1 to 15 cigarettes per day, (f) about 16 to 25 cigarettes per day, or (g) over 26 cigarettes per day. Groupings were based on a previous study of smoking among adolescents, ${ }^{26}$ with categories added for those who smoke less than daily.

\section{Nicotine dependence}

A version of the Fagerström tolerance questionnaire (FTQ) modified for use with adolescent smokers was used. ${ }^{26}$ The revised FTQ is a seven item questionnaire assessing factors associated with nicotine dependence. Possible scores range from 0 to 9 , with scores of 6 or higher indicative of substantial nicotine dependence.

\section{Stage of change}

To assess stage of change, participants were asked about their plans for smoking cessation, along with their history of quitting. ${ }^{27}$ Students who reported that they were not thinking of quitting in the next six months were classified as "precontemplators". "Contemplators" were those who were thinking about quitting smoking in the next six months. Students who were planning on quitting in the next 30 days were classified as being in the "preparation" stage. Participants who reported quitting within the past six months were considered to be in the "action" stage, while those who had been abstinent for more than six months were in the "maintenance" stage.

\section{Motivation to quit smoking}

Participants were asked at pretest how motivated they were not to smoke. A four point scale was used, with responses ranging from "not at all motivated" to "extremely motivated".

\section{Perceived benefits of smoking}

Beliefs about cigarette smoking were assessed at pretest and follow up using four items describing "benefits" commonly associated with smoking. Participants were asked to indicate on a four point scale how they felt smoking made a person look, with response options ranging from "very uncool" to "very cool". They also were asked whether they believed smoking helped them relax, the degree to which it aided concentration, and whether they thought it helped control weight. Response choices for these last three items were "not at all", "a little", "a fair amount", and "quite a bit".

\section{Social modelling}

Social modelling was assessed at pretest by having participants report how many of their family members and five closest friends were smokers. In addition, students were asked whether they felt pressure from their parents not to smoke ("not at all", "a little", "a fair amount", and "quite a bit").

\section{Knowledge about smoking}

Participants' knowledge about smoking was measured at pretest and post-test with a 12 item scale developed for this study. The questions were derived directly from the STS curriculum and were aimed at assessing the degree to which participants learned and retained the material presented in the programme. For example, one such item asked students how many ninth graders smoke daily. Response options included "most (about 80 out of 100 kids)", "half (about 50 out of 100 kids)", "some (about 25 out of 100 kids)", and "not many (about 6 out of 100 kids)". Our previous research in this region had established that the last response was correct. Another item asked students to identify when it was easiest to quit smoking, with response options including "when you have health problems", "when you are old", "when you are pregnant", and "when you are first starting to smoke". Each such question was individually scored as right or wrong, and the correct number of responses was summed, so that possible total scores on the measure ranged from $0-12$.

\section{Programme acceptability}

Several items were included at post-test to assess treated participants' impressions of the programme. For example, 
students were asked whether they felt the sessions were important ("very", "somewhat", "not at all"), how much they liked the programme ("liked it a lot", "liked it a little", "disliked it a little", "disliked it a lot"), and whether they would recommend the programme to a friend ("definitely", "maybe", "no"). In addition, at follow up participants were asked whether they would have sought help to quit smoking if they had not been offered the programme.

\section{Smoking cessation}

To be considered a quitter at post-test or follow up, students had to report abstinence from smoking for a period of at least seven days before the assessment. At post-test, the participants were initially randomly assigned to provide or not provide measures of alveolar CO. The CO measure was used as a "bogus pipeline" to encourage accurate reporting of smoking status. ${ }^{28}$ Because estimates of quitting appeared inflated, this procedure was later changed so that all students provided CO measures at post-test.

At follow up, all participants who reported quitting provided measures of $\mathrm{CO}$ and salivary cotinine. Cotinine concentrations of $\leqslant 15 \mathrm{ng} / \mathrm{ml}$ were used as the cut-off to classify participants as abstinent. ${ }^{29}$ When self reported smoking conflicted with biochemical measures, the results of the salivary cotinine analysis were used to determine quit status. Participants with missing cotinine data were conservatively classified as smokers.

\section{Data analysis}

Baseline comparisons between groups were conducted using $\chi^{2}$ tests of independence for categorical variables, and independent samples $t$ tests for continuous variables. Separate variance estimates were used as appropriate. Changes in knowledge and beliefs about smoking were assessed using 2 (condition) $\times 2$ (time) repeated measures analysis of variance (ANOVA). For the primary outcome of smoking cessation, differences between groups were investigated using logistic regression.

For analyses of differences between pretest and post-test, we used the 261 participants who completed post-test. We did not include the 22 students who dropped out of the programme, since dropouts tended to occur so early that significant treatment had not occurred. For example, $82 \%$ of those who dropped out of the intervention group during treatment attended two or fewer sessions. However, in our analysis of follow-up results, we used an intention-to-treat model, with any of the original 261 participants lost to follow up classified as smokers.

\section{RESULTS}

\section{Baseline characteristics}

Demographic characteristics of the 261 students who provided post-test data are presented in tables 1 and 2 . No significant differences were observed between the treatment and control groups on any of the baseline variables presented. The adolescents averaged almost 16 years of age, and most were male and white. The participants' parents usually had at least a high school education or equivalent (89\%). The students themselves more often came from grades 9 and 10 than grades 11 and 12. It may be that older students become more effective at avoiding getting caught smoking at school, or that they exit school as their smoking habit intensifies.

Participants' responses to questions about their family and friends' smoking habits suggest that these students were embedded in social environments that supported tobacco use. Fully $45 \%$ of these young smokers reported that all of their five closest friends smoke cigarettes regularly, and 32\% indicated that all of the family members they live with smoke. In addition, $52 \%$ of the participants indicated that they felt little pressure from their parents not to smoke, and $19 \%$ reported feeling no pressure at all.

Characteristics of the sample's tobacco use at baseline are presented in table 2. Most participants reported daily smoking, and the overwhelming majority had consumed at least 100 cigarettes in their lifetime. On average, students had smoked their first cigarette nearly four years earlier. More than $75 \%$ of the youth had made at least one quit attempt in the past year, and over $20 \%$ had tried to quit three or more times. Most of these adolescents demonstrated at least some symptoms of nicotine dependence, with nearly $20 \%$ of students scoring in the significantly dependent range on the FTQ. ${ }^{26}$ Despite their level of dependence and history of failed quit attempts, only $30 \%$ of the sample was in the precontemplation stage of change.

\section{Programme participation}

One measure of protocol success is the extent to which students continue in a research programme. Notably, only $8 \%$ of the 283 students who entered the research protocol dropped out before the post-test, and to date $91 \%$ of the eligible participants have completed the follow up (data collection is still continuing). After post-test, all remaining participants $(\mathrm{n}=261)$ students (both treatment and control) received monthly phone calls from their assigned health educator. Students in the treatment condition averaged (SD) 7.6 (2.6) sessions out of a maximum of 11 possible phone calls, compared to 6.9 (2.9) sessions completed by the controls ( $\mathrm{t}[148]=1.68, \mathrm{p}>0.10)$. Although we did not record the actual length of these calls, they were generally 3-5 minute conversations.

Our schools' administration certainly contributed to the retention over the life of this study by offering reduced suspensions for students participating in the study (regardless of treatment versus control condition). Students knew that if they dropped from the study the administration would revert to the usual procedure and introduce the full suspension instead. Once the post-test was complete, however, consequences for non-participation were less clear. For this reason, we provided incentives (for example, fast food coupons, nominal amounts of money, discounts to music stores) for students who completed their monthly calls and follow up assessment.

\section{Programme acceptability}

Responses to questions about the STS programme suggested that it was well received. For example, $89 \%$ of the students in the STS condition reported that they liked the programme, and approximately 95\% rated the sessions as "important" or "very important". In addition, 66\% of students reported that they would definitely recommend the programme to their friends. Despite their history of failed quit attempts, $66 \%$ of the students reported that they would not have sought help in the past year for quitting if they had not been offered the STS programme.

\section{Tobacco related knowledge}

To determine whether the treated students improved more than controls on knowledge of the curricular material, a repeated measures analysis was performed, with condition as a between groups variable. The main effect for condition was not significant, but there was a main effect for time ( $F[1$, $259]=42.06, \mathrm{p}<0.001)$, with scores for the sample as a whole improving from pretest to post-test. More importantly, a significant condition by time interaction emerged, indicating students in the intervention group improved in knowledge more than controls over the course of the programme $(F[1,259]=9.52, \mathrm{p}=0.002)$. 


\begin{tabular}{|c|c|c|}
\hline \multirow[b]{2}{*}{ Variable } & \multicolumn{2}{|c|}{ Proportion of sample (n) } \\
\hline & STS $(n=169)$ & Control $(n=92)$ \\
\hline \multicolumn{3}{|l|}{ Sex } \\
\hline Male & $62.1 \%(105)$ & $66.3 \%(61)$ \\
\hline Female & $37.9 \%(64)$ & $33.7 \%(31)$ \\
\hline \multicolumn{3}{|l|}{ Race/ethnicity } \\
\hline African American & $11.8 \%(20)$ & $15.2 \%(14)$ \\
\hline White & $81.7 \%(138)$ & $81.5 \%(75)$ \\
\hline Other & $6.5 \%(11)$ & $3.3 \%(3)$ \\
\hline \multicolumn{3}{|l|}{ Grade } \\
\hline 7 & $1.8 \%(3)$ & $1.1 \%(1)$ \\
\hline 8 & $5.9 \%(10)$ & $4.3 \%(4)$ \\
\hline 9 & $26.0 \%(44)$ & $29.3 \%(27)$ \\
\hline 10 & $29.6 \%(50)$ & $30.4 \%(28)$ \\
\hline 11 & $22.5 \%(38)$ & $18.5 \%(17)$ \\
\hline 12 & $14.2 \%(24)$ & $16.3 \%(15)$ \\
\hline \multicolumn{3}{|c|}{ Highest educational obtainment of parent/guardian } \\
\hline Less than high school & $12.5 \%(21)$ & $8.7 \%(8)$ \\
\hline High school diploma or equivalent & $25.6 \%(43)$ & $28.3 \%(26)$ \\
\hline Some college & $21.4 \%(36)$ & $17.4 \%(16)$ \\
\hline College degree & $40.5 \%(68)$ & $45.7 \%(42)$ \\
\hline
\end{tabular}

\section{Beliefs about the "benefits" of smoking}

Repeated measures analyses were also used to detect differences in treatment versus control group changes in attitudes from pretest to follow up. We first examined shifts in the belief that smoking reduces weight. Analysis revealed that the main effect for time was marginally significant ( $F[1$, $119]=3.78, \mathrm{p}=0.054)$, with both groups tending to endorse this belief less at follow up than at pretest. The main effect for condition and the condition by time interaction were both non-significant.

Next we explored changes in students' beliefs that smoking would enhance their social image. A significant main effect for time emerged $(F[1,93]=7.82, \mathrm{p}=0.006)$, with both groups becoming less convinced by follow up that smoking would make them look "cool". There was no significant main effect for condition, and the condition by time interaction was not reliable.
Changes in the belief that smoking aids relaxation were also examined. Analysis did not reveal any main effects or interactions.

Finally, when we examined the belief that smoking aids concentration, we found a significant main effect for time, such that participants (regardless of group assignment) were less likely to endorse this belief at follow up than at pretest $(F$ $[1,124]=5.17, \mathrm{p}=0.03)$. Neither the main effect for condition nor the condition by time interaction was significant.

\section{Smoking cessation}

Post-treatment

Participants demonstrated similar rates of quitting over time regardless of group assignment. Overall, $20.1 \%$ of students in the STS group and $24.2 \%$ of those in the control group reported abstinence at post-test (odds ratio (OR) 0.79,

\begin{tabular}{|c|c|c|}
\hline \multirow[b]{2}{*}{ Variable } & \multicolumn{2}{|c|}{ Proportion of sample (n) } \\
\hline & STS $(n=169)$ & Control $(n=169)$ \\
\hline Smoked $100+$ cigarettes in lifetime & $82.0 \%(137)$ & $80.2 \%(73)$ \\
\hline \multicolumn{3}{|l|}{ Smoking frequency } \\
\hline Less than weekly smokers & $14.2 \%(24)$ & $13.0 \%(12)$ \\
\hline Weekly smokers & $18.9 \%(32)$ & $15.2 \%(14)$ \\
\hline Daily smokers & $66.9 \%(113)$ & $71.7 \%(66)$ \\
\hline $1-15$ cigarettes/day & $50.9 \%(86)$ & $56.5 \%(52)$ \\
\hline $16-25$ cigarettes/day & $11.2 \%(19)$ & $14.1 \%(13)$ \\
\hline $26+$ cigarettes/day & $4.7 \%(8)$ & $1.1 \%(1)$ \\
\hline \multicolumn{3}{|l|}{ Modified Fagerström scores } \\
\hline $0-2$ & $25.4 \%(43)$ & $29.7 \%(27)$ \\
\hline $3-5$ & $53.3 \%(90)$ & $54.9 \%(50)$ \\
\hline$\geqslant 6$ & $21.3 \%(36)$ & $15.4 \%(14)$ \\
\hline \multicolumn{3}{|l|}{ Stage of change } \\
\hline Precontemplation & $31.0 \%(52)$ & $28.6 \%(26)$ \\
\hline Contemplation & $26.8 \%(45)$ & $35.2 \%(32)$ \\
\hline Preparation & $15.5 \%(26)$ & $12.1 \%(11)$ \\
\hline Action & $26.8 \%(45)$ & $24.2 \%(22)$ \\
\hline \multicolumn{3}{|l|}{ Number of quit attempts in past year } \\
\hline None & $23.7 \%(40)$ & $23.9 \%(22)$ \\
\hline One & $24.9 \%(42)$ & $39.1 \%(36)$ \\
\hline Two & $29.6 \%(50)$ & $16.3 \%(15)$ \\
\hline Three or more & $21.9 \%(37)$ & $20.7 \%(19)$ \\
\hline
\end{tabular}


$\mathrm{p}=0.45)$. Among those for whom CO measures were not obtained, self reported cessation rates were $24.2 \%$ for the treatment group, compared to $30.2 \%$ among controls (OR $0.74, p=0.38$ ). For those whose self reports were obtained under bogus pipeline conditions (that is, CO measures obtained), self reported cessation rates were $10.2 \%$ in the treatment group and $10.7 \%$ for controls (OR 0.95, p $=0.94$ ). Thus, self reported cessation rates were two to three times higher when the self report was obtained without a pipeline. The over reporting of cessation occurred in both treatment and control groups, but the degree of inflation when no pipeline was in place appeared to be greater among those in the control condition.

\section{Twelve month follow up}

At follow up, $26.3 \%$ of the STS group and $27.5 \%$ of control participants self reported that they had quit smoking (OR $0.94, \mathrm{p}=0.88)$. For all participants who reported abstinence, biochemical verification of smoking status was attempted. Salivary cotinine samples were successfully obtained from 18 of the 41 students who reported cessation. Results of the cotinine analyses indicated that $50 \%$ of those who reported quitting had falsified their smoking status (that is, had cotinine values of $>15 \mathrm{ng} / \mathrm{ml}$ ). Falsification rates were $40 \%$ and $63 \%$ for participants in the STS and control groups, respectively. Thus, it appeared that control participants more readily falsified their self reports than students who had undergone treatment. To obtain a more conservative measure of cessation, we then considered students to be abstinent only when their self reports had been biochemically verified. Under these conditions, both the treatment and control groups obtained only $6 \%$ cessation at the one year follow up.

Given the small number of abstinent teens at follow up, we lacked the power to conduct formal analysis to determine how they differed at pretest from those who continued to smoke. However, we did observe that participants who were confirmed abstinent at follow up had been relatively light smokers with little dependence. For example, the mean (SD) score on the modified FTQ was 1.67 (0.71) (range 0-3). Fifty six per cent of the abstinent participants had smoked less than daily, with none smoking more than 15 cigarettes per day. Forty two per cent of the quitters had been smoking for less than one year. Sixty seven per cent reported that they had tried to quit smoking at least once during the past year. Notably, all of the participants who were confirmed abstinent at follow up were classified as being in the action stage of change at baseline. However, these characteristics were not necessarily predictive of treatment responsiveness. For example, there was no evidence that the treatment was any more effective with students in more advanced stages of change at pretest than those who were in precontemplation when they entered the study $(\mathrm{p}>0.05)$.

\section{DISCUSSION}

To our knowledge, this is only the second investigation to recruit students caught with cigarettes at school. ${ }^{30}$ In our experience, the approach is highly successful. Eighteen high schools were recruited without difficulty into this investigation, and the cessation programme fit into the school procedures seamlessly, despite the demands of the research protocol. School personnel clearly recognised the need for something other than a punitive approach to smoking. Their willingness to reduce punitive sanctions for students who entered the programme was integral to the programme's success.

Given this level of school support, it should not be surprising that student enrolment actually exceeded our expectations. Fully $83 \%$ of the adolescents offered the research programme agreed to enrol. Further, most of these students were daily smokers with at least one previous attempt to quit smoking. At the time of their referral, $70 \%$ were at least contemplating another quit attempt. Thus, this recruitment method produced considerable reach into the population of daily smokers who were receptive to quitting smoking.

Retention throughout the life of the programme was also much higher than most studies in this area. Fully $92 \%$ of those who entered the study completed post-test, and although we are still collecting follow up data, to date we have a $91 \%$ completion rate for students who have reached the one year mark. Perhaps one reason for this high retention rate is that the programme itself was carefully designed to be appealing to young people. Programme evaluation data indicated that by far the majority of students viewed the programme as important and would recommend it to a friend. Notably, most of these young smokers also reported that they would not have sought help quitting if the programme had not been offered. As difficult as it is to reach adolescent smokers, these data argue that the effort to make cessation programmes available is critical, because teens are unlikely to seek help on their own.

Despite our success in reaching and retaining adolescent smokers for this investigation, our outcomes were disappointing. Treated students did learn the curricular material, but at follow up their attitudes toward smoking had not improved relative to those of untreated controls. Worse yet, there were no significant differences between the treated and control youth on quit rates at post-test or at follow up.

As disappointing as our findings are, they highlight several methodological issues that must be addressed in this literature. First, our strategy of randomising within schools may have interfered with the detection of changes in attitudes, if not behaviours. Recall that treatment and control participants co-existed in the same schools. Especially since they liked the intervention, the treatment students may well have talked about the programme content with the control students, so that eventually both groups' attitudes became more negative toward tobacco. Such a phenomenon could explain why the groups showed no significant differences between attitudes but changed in a like manner over time. Randomising schools rather than students would make it less likely that this type of contamination would occur.

Second, our investigation illustrates why control groups are critical. In their review, ${ }^{18}$ Sussman and colleagues concluded that the average post-treatment cessation rate was $21 \%$. Our study yielded a similar overall post-test cessation rate for treated students $(20 \%)$. However, the quit rate for our control group was slightly higher (24\%). These findings should remind us that solid methodology requires randomisation with a control group, before the effects of any treatment programme can be properly gauged.

A similar issue arises when measurement methods are considered. Most previous studies ${ }^{31}{ }^{32}$ have relied on selfreports of quitting, without a bogus pipeline in place. In this situation, the validity of quit rates cannot be established. To estimate the effect of measurement method on outcome, we collected self reports from some participants, but used a bogus pipeline for others. The results were notably different. Under self report conditions, both the treatment and control groups had unusually high reported quit rates. However, the control group's quit rate was systematically more inflated than that of the treatment group. Under bogus pipeline conditions, the reported quit rates for treatment and control groups were much more conservative and comparable to each other.

An almost identical pattern emerged at follow up. We found that over one quarter of both treated and control participants self reported cessation. However, salivary cotinine 
measures suggested high rates of falsification. Further, the falsification rate appeared higher for controls. When only biochemically verified abstinence was considered, quit rates fell to $6 \%$ for both treatment and control groups. This rate is remarkably similar to that of another study providing a one year follow up of an adolescent cessation programme. ${ }^{33}$

The reasons that students might inflate their quit rates less when they are in a treatment group are not entirely clear. However, allegiance to health educators may play a role. Over the course of the study, participants typically develop a close relationship with the health educator assigned to them. Students may find it more difficult to lie about their smoking when they have an established relationship with any member of the research team. A similar effect has been identified by researchers assessing smoking prevention programme outcomes. ${ }^{34}$ The net effect of this pattern is that treatment effects are more likely to be underestimated when no validation procedure is used. These findings should serve to caution researchers about relying on self reports of adolescent cessation alone. By failing to use a pipeline procedure or biochemical validation, they may increase the likelihood that they could miss genuine treatment effects.

Despite its strengths, a number of limitations of the current study should be addressed. First, our randomisation of students within schools might have allowed for some contamination of groups; that is, treated students might have shared information with untreated controls. If this occurred and had a meaningful effect, it might account in part for the lack of significant differences between groups. A better alternative would be to recruit a large number of schools and randomise those to conditions. Second, it should be noted that this was a select population of students who were not necessarily seeking treatment, and our negative results may not generalise to a population of students presenting for help with smoking cessation. Finally, our intervention was drawn primarily from successful adult smoking cessation treatments, which may not be developmentally suited for teens.

How might cessation programmes be better adapted for adolescents? One obvious recommendation is that we explore the social consequences of quitting for teens. Our results indicate the students referred for quit programmes are often embedded in a community of smokers, and the odds of success decline when those close to teens smoke. ${ }^{13} 18$ Nonetheless, there is essentially no research on the social reactions adolescents experience when they attempt to quit. It may be that smoking parents and friends sometimes react in an actively hostile fashion. Even family and friends who want to help may have no idea what to expect during a quit attempt, and symptoms of withdrawal may be very difficult for family and friends to tolerate. Research assessing the attitudes of parents and close friends toward quit attempts, their expectations regarding withdrawal symptoms, and their reactions over the course of a quit attempt might help us construct a picture of the social consequences of quitting for teens. With this knowledge, we might begin to develop more realistic ways to help adolescents cope with the social changes their quitting may trigger.

Second, we must acknowledge that withdrawal effects are a major issue, even among adolescents who are relatively light smokers. ${ }^{35}$ Only recently have prospective studies ${ }^{36}$ been conducted to examine withdrawal among teen smokers, but numerous researchers ${ }^{37}{ }^{38}$ have emphasised that pharmacotherapy to assist adolescents in quitting should be considered. Fortunately, a number of trials are currently underway to evaluate the efficacy of nicotine replacement therapy and other pharmacologic approaches to withdrawal, and their results may help identify at least a subset of adolescents who are responsive to these treatments.

\section{What this paper adds}

To date, few cessation programmes targeting adolescents have been developed and evaluated, and the investigations that have been conducted have suffered from serious methodological flaws (for example, few control groups, reliance on self reports of smoking status rather than biochemical verification, short follow ups, and high dropout rates). The purpose of the current study was to provide a well designed randomised controlled trial of a smoking cessation programme for youth that eliminated these limitations.

Although we were successful in maintaining the integrity of the research protocol in a school setting, the results of the programme were disappointing. We failed to find any evidence that the treatment programme produced greater cessation than that which occurred naturally in the control group. However, several central methodological findings emerged. First it was clear that control groups are critical in studies of this kind, because quit rates tend to be quite inflated. Second, this inflation occurs more among youth in control conditions; thus, cessation programmes constitute one situation in which biochemical verification rather than self reports must be employed to measure smoking status.

Third, considering the previous failures of nonpharmacologic approaches to teen cessation, we should review our basic assumptions about how cessation programmes for adolescents should be constructed. These programmes have generally been built using adult programmes as models, ${ }^{39}$ but many of the components that make adult programmes successful may not apply to youth. ${ }^{40}$ It may be more helpful to dismantle the components commonly thought useful in adolescent cessation programmes and carefully evaluate them individually, to determine whether specific components have the intended effect on adolescent smokers. In this way, we might begin to understand what is useful in the complex programmes we are presenting to young smokers-and what is not. Further, we may need to carefully consider the individual characteristics of the adolescent when prescribing cessation programmes. For example, matching the intervention to the youth's stage of change may reduce resistance and improve outcome.

Finally, we may need to reconsider our expectations for adolescent smoking cessation programmes. It has been estimated that the average adult who successfully quits smoking does so after five to six failed attempts. Apparently, even adults need to go through a process of quitting and relapsing before they learn sufficient skills to overcome the addiction. However, adolescents have not yet achieved the maturity of adults, including the ability to set clear goals, think through anticipated difficulties, settle on a specific plan, and utilise social and other resources for achieving goals. Thus, the low success rates from adolescent cessation programmes may reflect the developmental limitations of the population under study.

Even if developmental issues interfere with programme success, cessation programming for teens is not necessarily futile. Our goals, however, may need to change. Perhaps over the course of these programmes, participants gradually learn skills that crystallise somewhat later in adolescence. If so, the programmes may prime participants for repeated efforts to quit, and the eventual positive effect of the programmes may be demonstrated later in adolescence, when the teens' maturity increases. In this case, only studies with longer follow ups would be able to detect this type of delayed treatment response. We hope that as research in this field 
grows, investigators will conduct longer term studies, examining the many ways that cessation programmes could change the trajectory of tobacco use among adolescent smokers.

\section{ACKNOWLEDGEMENTS}

This study was supported by a grant (DA12532) from the National Institute on Drug Abuse. We are especially grateful to the administration, faculty, and students of the Memphis City and Shelby County schools for their support of this research.

\section{Authors' affiliations}

L A Robinson, The University of Memphis Department of Psychology, Memphis, Tennessee, USA

M W Vander Weg, R C Klesges, B McLain-Allen, The University of

Memphis Center for Community Health

B W Riedel, The Memphis City Schools Office of Research and Evaluation

\section{REFERENCES}

1 Centers for Disease Control. Trends in cigarette smoking among high school students - United States, 1991-1999. MMWR Morb Mortal Wkly Rep 2000:49:775-8

2 US Department of Health and Human Services. Preventing tobacco use among young people. A report of the Surgeon General, 1994. Atlanta, Georgia: Public Health Service, Centers for Disease Control and Prevention Office on Smoking and Health, 1994. (US Government Printing Office Publication No S/N 017-001-00491-0.)

3 Chassin L, Presson CC, Sherman SJ, et al. The natural history of cigarette smoking: predicting young-adults smoking outcomes from adolescent smoking patterns. Health Psychol 1990;9:701-16.

4 Centers for Disease Control. Projected smoking-related deaths among youth-United States. MMWR Morbid Mortal Wkly Rep 1996;45:971-4.

5 Ershler J, Leventhal H, Fleming R, et al. The quitting experience for smokers in sixth through twelfth grades. Addict Behav 1989;14:365-78.

6 Burt RD, Peterson AV. Smoking cessation among high school seniors. Prev Med 1998;27:319-27.

7 Friestad C, Rise J. Smoking attributions and adolescents' intention to try to quit smoking. Addict Res 1998;6:13-26

8 Stone SL, Kristeller JL. Attitudes of adolescents toward smoking cessation. Am J Prev Med 1992:8:221-5.

9 Riedel BW, Robinson LA, Klesges RC, et al. What motivates adolescent smokers to make a quit attempt? Drug Alcohol Depend 2002;68:167-74.

10 Gillespie A, Stanton W, Lowe JB, et al. Feasibility of school-based smoking cessation programs. J Sch Health 1995:65:432-7.

11 Stanton WR, McClelland M, Elwood C, et al. Prevalence, reliability and bias of adolescents' reports of smoking and quitting. Addiction 1996;91:1705-14.

12 Riedel BW, Robinson LA, Klesges RC, et al. Characteristics of adolescents caught with cigarettes at school: implications for developing smoking cessation programs. Nicotine Tob Res 2002:4:351-4

13 Sussman S, Dent CW, Severson $H$, et al. Self-initiated quitting among adolescent smokers. Prev Med 1998;27:A19-28.

14 Colby SM, Tiffany ST, Shiffman S, et al. Are adolescent smokers dependent on nicotine? A review of the evidence. Drug Alcohol Depend 2000;59/suppl 1):S83-95.

15 Sussman S. School-based tobacco use: prevention and cessation: where are we going? Am J Health Behav 2001;25:191-9.
16 Pallonen VE, Murray DM, Schmid L, et al. Patterns of self-initiated smoking cessation among young adults. Health Psychol 1990;9:418-26.

$17 \mathrm{McNeill}$ AD. The development of dependence on smoking in children. Br J Addict 1991:86:589-92.

18 Sussman S, Lichtman K, Ritt A, et al. Effects of thirty-four adolescent tobacco use cessation and prevention trials on regular users of tobacco products. Subst Use Misuse 1999;34:1469-503.

19 Colby SM, Monti PM, Barnett NP, et al. Brief motivational interviewing in a hospital setting for adolescent smoking: a preliminary study. J Consult Clin Psych 1998;66:574-8.

20 Fagan $P$, Frazier L, Sorensen G. The SMART teens against the risks of tobacco project: A worksite-based methods development study. Paper presented at the $\mathrm{NCl}$ Tobacco Use Among Youth Research Investigators Meeting. Park City, Utah, 2001.

21 Hanson K, Hatsukami D, Jenson S, et al. Smoking cessation among teenagers using the nicotine patch. Poster presented at the Society for Research on Nicotine and Tobacco's Seventh Annual Scientific Sessions. Seattle, 2001.

22 Adelman WP, Duggan AK, Hauptman P, et al. Effectiveness of a high school smoking cessation program. Pediatrics 2001;107:1-8.

23 Sussman S, Dent CW, Lichtman KL. Project EX: outcomes of a teen smoking cessation program. Addict Behav $2001 ; 26: 425-38$.

24 Miller WR, Rollnick S. Motivational interviewing. New York: Guilford Press, 1991.

25 Prochaska JO, DiClemente CC. Stages and process of self-change of smoking: Toward an integrative model of change. J Consult Clin Psych 1983;51:390-5.

26 Prokhorov AV, Pallonen UE, Fava JL, et al. Measuring nicotine dependence among high-risk adolescent smokers. Addict Behav 1996;21:117-27.

27 Pallonen UE. Transtheoretical measures for adolescent and adult smokers: similarities and differences. Prev Med 1998;27:A29-38.

28 Murray DM, O'Connell CM, Schmid LA, et al. The validity of smoking selfreports by adolescents: a re-examination of the bogus pipeline procedure. Addict Behav 1987;12:7-15.

29 Benowitz NL, Jacob P, Ahijevych K, et al. Biochemical verification of tobacco use and cessation. Nicotine Tob Res 2002;4:149-59.

30 Coleman-Wallace D, Lee JW, Montgomery S, et al. Evaluation of developmentally appropriate programs for adolescent tobacco cessation. J Sch Health 1999:69:314-19.

31 Pendell WK. Community intervention. Intervening with teen tobacco users. In: Tobacco Education Group (TEG). Minneapolis, Minnesota: Community Intervention, Inc, 1996.

32 Prince $\mathbf{F}$. The relative effectiveness of a peer-led and adult-led smoking intervention program. Adolescence 1995;30:187-94.

33 Smith TA, House RF, Croghan IT, et al. Nicotine patch therapy in adolescent smokers. Pediatrics 1996:98:659-67.

34 Komro KA, Kelder SH, Perry CL, et al. Effects of a saliva pipeline procedure on adolescent self-reported smoking behaviour and youth smoking prevention outcomes. Prev Med 1993;22:857-65.

35 Difranza JR, Rigotti NA, McNeill AD, et al. Initial symptoms of nicotine dependence in adolescents. Tobacco Control 2000:9:313-19.

36 Riedel BW, Robinson LA, Klesges RC, et al. Ethnic differences in smoking withdrawal effects among adolescents. Addict Behav 2003;28:129-40.

37 Hurt RD, Croghan GA, Beede SD, et al. Nicotine patch therapy in 101 adolescent smokers: Efficacy, withdrawal symptom relief, and carbon monoxide and plasma cotinine levels. Arch Ped Adol Med 2000;154:31-7.

38 Prokhorov AV, Judmon KS, de Moor CA, et al. Nicotine dependence, withdrawal symptoms, and adolescents' readiness to quit smoking. Nicotine Tobacco Res 2001;3:151-5.

39 Lamkin L, Davis B, Kamen A. Rationale for tobacco cessation interventions for youth. Prev Med 1998;27:A3-8.

40 Henningfield JE, Michaelides T, Sussman S. Developing treatment for tobacco addicted youth-Issues and challenges. J Child Adol Subst Abuse 2000;9:5-26. 